\title{
RESEARCH
}

Open Access

\section{Validity of the French version of Catquest- 9SF and use of an electronic notepad for entering patient-reported outcome measures}

Gregory Katz ${ }^{1,2,3}$, Alexandra Rouquette ${ }^{4,5}$, François Lignereux 6 , Thierry Mourgues ${ }^{7}$, Michel Weber ${ }^{8}$ and Mats Lundström ${ }^{9 *}$ (D)

\begin{abstract}
Background: The Catquest-9SF questionnaire is a patient reported outcome measure that quantifies the visual benefits from cataract surgery. The purpose of this study was to translate and adapt the Catquest-9SF questionnaire for France, to assess its psychometric properties via Rasch analysis, and to assess its validity when completed using an electronic notepad.
\end{abstract}

Methods: The Catquest-9SF questionnaire was translated following the guidelines of the International Society for Pharmacoeconomics and Outcomes Research. Catquest-9SF and clinical data were collected from patients before and after routine cataract surgery. All questionnaire data were collected via an electronic notepad. Rasch analysis was performed to assess psychometric properties, and sensitivity to change was analysed for patients with complete paired pre- and post-operative questionnaires.

Results: A complete filled-in preoperative questionnaire was obtained for 848 patients. Rasch analysis showed good precision (person separation: 2.32, person reliability: 0.84 ), ordered category probability curves, no item misfit, and unidimensionality. The respondents were slightly more able than the level of item difficulty (targeting: -1.12 logits). Sensitivity was analysed on 211 paired questionnaires, and the postoperative questionnaires showed a clear ceiling effect. The effect size was 2.6. The use of an electronic notepad for completing the questionnaire worked out very well after some adjustments.

Conclusions: The French version of Catquest-9SF has good psychometric properties and is suitable for use in French-speaking patients. The use of the Catquest-9SF questionnaire in an electronic format showed good validity.

Keywords: Cataracts, Patient-related outcome measures, Quality of life, Questionnaire, Value-based health care, Catquest-9SF

\footnotetext{
* Correspondence: mats.lundstrom@karlskrona.mail.telia.com

${ }^{9}$ Department of Clinical Sciences, Ophthalmology, Faculty of Medicine, Lund University, Lund, Sweden

Full list of author information is available at the end of the article
}

(c) The Author(s). 2021 Open Access This article is licensed under a Creative Commons Attribution 4.0 International License, which permits use, sharing, adaptation, distribution and reproduction in any medium or format, as long as you give appropriate credit to the original author(s) and the source, provide a link to the Creative Commons licence, and indicate if changes were made. The images or other third party material in this article are included in the article's Creative Commons licence, unless indicated otherwise in a credit line to the material. If material is not included in the article's Creative Commons licence and your intended use is not permitted by statutory regulation or exceeds the permitted use, you will need to obtain permission directly from the copyright holder. To view a copy of this licence, visit http://creativecommons.org/licenses/by/4.0/ The Creative Commons Public Domain Dedication waiver (http://creativecommons.org/publicdomain/zero/1.0/) applies to the data made available in this article, unless otherwise stated in a credit line to the data. 


\section{Background}

Cataract surgery is one of the most frequently performed surgical procedures and has low complication rates [1]. While objective measures such as visual acuity and residual refractive error are critical results from the surgeon's perspective, the ability to perform day-to-day tasks is an essential outcome from the patient's perspective. Some patients who have undergone uneventful cataract surgery with gains in visual acuity may still not be satisfied [2]. This can be due to a variety of reasons, such as patient expectations, or because visual acuity does not reflect the patient's quality of life, ability loss, or limitations in daily activities [3]. In addition, the mean waiting time for cataract surgery in OECD countries is 129 days [4], which can cause dissatisfaction and frustration, and strengthens the need to assess patients' quality of life.

Several patient-reported outcome questionnaires have been developed to quantify the subjective visual function in cataract patients [5]. The Catquest-9SF questionnaire is one such example, measuring activity limitations in daily life for patients with cataract or following cataract surgery [6]. It comprises nine questions: two global items evaluating the patient's general perception of difficulties and satisfaction with vision, and seven items related to difficulties in performing specific daily activities [6]. Each item has four response options ranging from 1 (very great difficulty) to 4 (no difficulty), and a "cannot decide" option which is treated as a missing answer. A raw score ranging from 9 to 36 is computed in summing the answers to the 9 items. Catquest-9SF has demonstrated robust psychometric properties, and when compared to 15 other Rasch-refined cataract questionnaires it proved to be the most responsive to cataract surgery [7].

The European Registry of Quality Outcomes for Cataract and Refractive Surgery (EUREQUO) database uses Catquest-9SF to assess cataract outcomes and develop evidence-based guidelines for cataract and refractive surgery in Europe [8]. The International Consortium for Health Outcomes Measurement (ICHOM) has adopted Catquest-9SF as a patient-reported outcome measure for cataracts to facilitate international comparisons that can drive improvements in patients' functional recovery and quality of life [9]. The questionnaire is available in Swedish [10], Australian English [11], German [12], Italian [13], Spanish [14], Dutch [15], Danish [16], and Chinese [17], and is currently being translated into several other languages.

The purpose of the present study was twofold: first, to translate and culturally adapt the Catquest-9SF questionnaire into French and to assess its psychometric properties via Rasch analysis; and second, to assess the validity of Catquest-9SF when completed using electronic notepads. Until now, most patients have used pencil and paper to complete the Catquest-9SF questionnaire, after which the data are entered into a spreadsheet. This spreadsheet data entry is not only tedious and time-consuming, but also carries the risk of typographical errors affecting data quality. These difficulties have stifled the adoption of the Catquest-9SF in daily clinical practice. Validation of an electronic method would enable the immediate use of the patient-reported outcomes during consultations.

\section{Methods \\ Translation}

The Catquest-9SF questionnaire was translated from the English version into French by Mapi Language Services (Lyon, France). To optimize both semantic and conceptual equivalence between the source and target language versions, the ten steps of the International Society for Pharmacoeconomics and Outcomes Research (ISPOR) principles of good practice for the translation and cultural adaption process for patient-reported outcomes were adopted [18]. These steps were: preparation, two parallel independent forward translations from English to French, reconciliation, backward translation, backward translation review, harmonization, cognitive debriefing with five native French speakers who had undergone cataract surgery, review of the cognitive debriefing results and finalization, proofreading results and the final report.

\section{Data collection}

Following the French translation of the Catquest-9SF, patients undergoing cataract surgery from February 2018 to August 2019 in two reference centres in France (Institut Ophtalmologique Sourdille-Atlantique in Nantes, and Ophtalmologie Chénieux at the Polyclinique de Limoges) were invited to complete the questionnaire before surgery and within 3 months following surgery. Patients were instructed by their surgeon to fill in the postoperative questionnaire after wearing their new spectacles (if any), and to schedule a postoperative visit when they were used to their new visual status. Exclusion criteria included difficulty with the French language or comprehension, being under guardianship or curatorship, and being under 18 years of age. All patients had a complete ophthalmic examination before and after surgery, and the following data were collected: age, gender, uncorrected distance visual acuity, spherical equivalent refraction, and spectacle-corrected distance visual acuity.

Information about the questionnaire was provided to the patients through the notepad. The patients were only able to access the questionnaire after explicitly confirming that they were not opposed to the scientific use of the data collected through the questionnaire. The 
A. Estimez-vous que votre vue occasionne actuellement des difficultés dans votre vie quotidienne?

\begin{tabular}{|c|c|c|c|c|}
\hline $\begin{array}{l}\text { Oui, de très } \\
\text { grandes } \\
\text { difficultés }\end{array}$ & $\begin{array}{c}\text { Oui, de } \\
\text { grandes } \\
\text { difficultés }\end{array}$ & $\begin{array}{l}\text { Oui, de légères } \\
\text { difficultés }\end{array}$ & $\begin{array}{l}\text { Non, aucune } \\
\text { difficulté }\end{array}$ & Je ne sais pas \\
\hline$\sqcup$ & 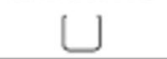 & 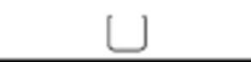 & 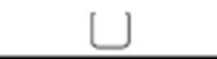 & $\sqcup$ \\
\hline
\end{tabular}

B. Actuellement, êtes-vous satisfait ou insatisfait de votre vue?

Très
insatisfait $\begin{gathered}\text { Plutôt } \\ \text { insatisfait }\end{gathered}$

C. Votre vue vous occasionne-t-elle des difficultés pour les activités suivantes?

Si oui, dans quelle mesure ? Sur chaque ligne, veuillez cocher la case qui vous parait correspondre le mieux à votre situation.

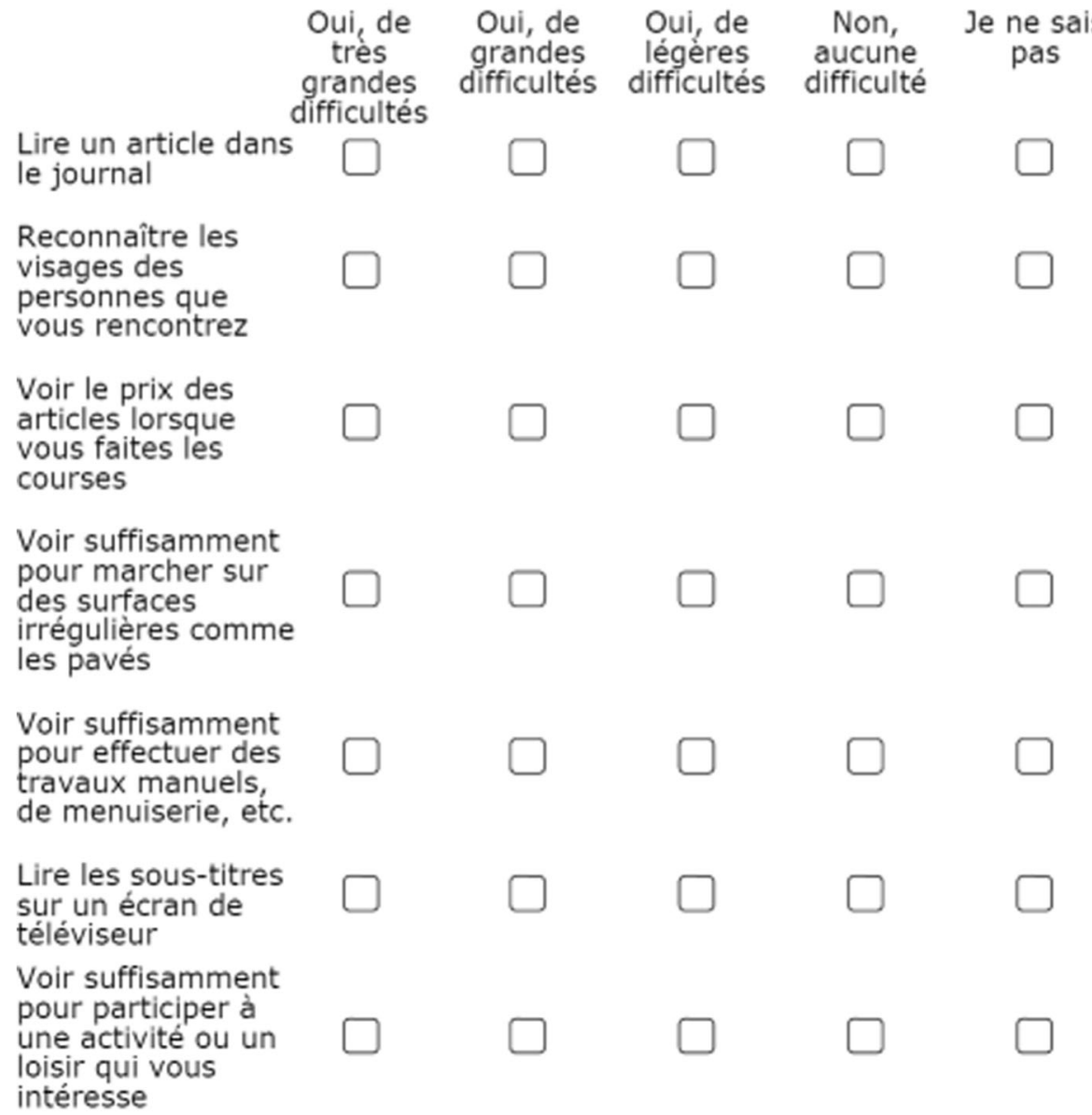

Merci beaucoup pour votre participation.

Catquest - FrancelFrench - Version of 30 Jan 17 - Mapi.

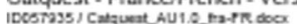

Fig. 1 French version of Catquest-9SF

dissemination of the questionnaire was systematic, and was part of routine care. These prospectively collected data were used to assess the psychometric properties of the questionnaire.
A declaration to the French data protection authority was registered in 2017 to comply with data pseudonymization and the General Data Protection Regulation (GDPR). 


\section{Digital notepad}

The French version of Catquest-9SF is shown in Fig. 1.

The French version of the Catquest-9SF was integrated into a digital application specifically developed by Value2Health for cataract patients, with one question per screen (Fig. 2). The ergonomic design included a simple layout with large buttons to enable self-reported assessment. All data collection was performed on an electronic notepad. The workflow description of using the notepad can be found in the online supplemental material.

The result of a completed Catquest-9SF is visible on the practitioner's dashboard (Fig. 3).

\section{Statistical analyses}

Analyses were performed on pre-surgical data except for the study of sensitivity to change which used paired preand post-surgical data. To be included in the analyses, patients had to respond to both general items (A and B) and at least five of the remaining seven $(\mathrm{C} 1-\mathrm{C} 7)$ Catquest-9SF items. Continuous and categorical variables were described as median (1st quartile-3rd quartile) and count (percentage) respectively. Descriptive analyses were conducted using version 15 of Stata [19], and Rasch analysis was performed using version 4.2.0 of Winsteps (Chicago, IL, USA).

Ceiling and floor effects were identified for each item of the Catquest-9SF; these two effects were defined a priori as more than $95 \%$ of respondents selecting the highest and the lowest category, respectively [20]. At the scale level, floor and ceiling effects were considered if more than $15 \%$ of respondents achieved the lowest or higher possible score, respectively [21]. Reliability was assessed using ordinal Cronbach's alpha [22] and considered satisfactory if $\geq 0.7$ [23]. Sensitivity to change was evaluated using a two-sided paired t-test comparing post- and pre-surgical Catquest-9SF raw scores, with a type- 1 error risk set at 0.05 . The effect size was also calculated based on Rasch scores.

The characteristics of the Catquest-9SF data were assessed by Rasch analysis, which is based on modern test theory and results in the transformation of an ordinal raw score into an interval Rasch score [24]. This means that the respondents' ability and the difficulty of the items are evaluated along the same scale. The unit of the scale is the logit, and so both respondents and items are given a logit value which constitutes their Rasch score. The distance in logits between one respondent and one item reflects the probability that the respondent will endorse the item. This probabilistic relationship is the basis for the estimation of interval scores.

Rasch analysis provides extensive insight into the psychometric properties of a questionnaire. This study assessed measurement precision in terms of person separation ( $>2.0$ considered adequate) and separation reliability ( $>0.80$ considered adequate), whether response categories are utilized in ascending order (category ordering with category probability curves), item fit to the model (outfit and infit statistics, with $0.70-1.30$ considered ideal and 0.50-1.50 acceptable), and whether the scale measures one or more dimensions (i.e., dimensionality; for unidimensionality, $\geq 60 \%$ of variance explained by the measure is ideal, while $\geq 50 \%$ is acceptable; the eigenvalue of the first contrast should be $<2.0$ ). Assessment of psychometric performance followed the standards of Khadka et al. [5].

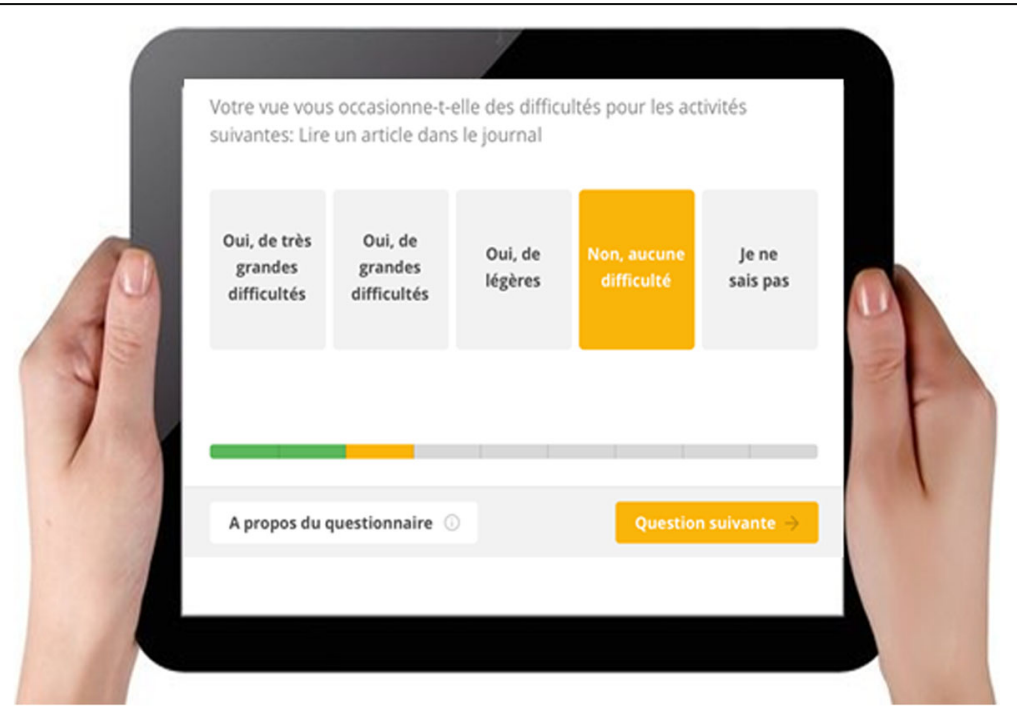

Fig. 2 Digital version of the French Catquest-9SF. One question is displayed per screen and large buttons are used for responses 


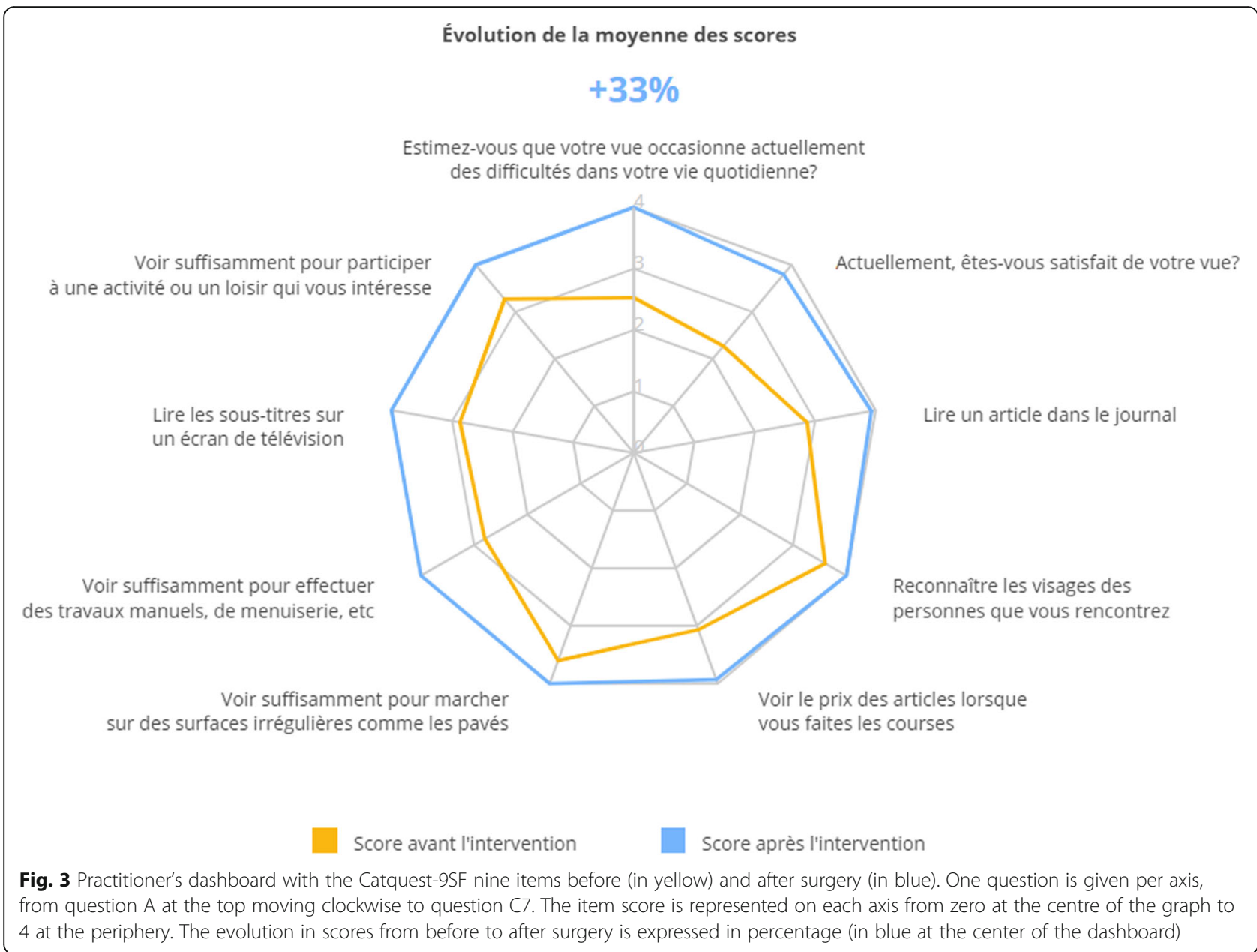

\section{Results}

Of the 894 patients included during the 18 -month recruitment period, 848 (331 males, 39\%) responded to items $\mathrm{A}$ and $\mathrm{B}$ and at least 5 of the remaining 7 Catquest-9SF items before the surgery. Their median age was $71.9(67.6-78.2)$ years. One-eye surgery was performed in 388 (46\%) of these patients while the remainder underwent both-eye surgery. The distribution of the pre-surgical responses to the nine Catquest-9SF items is shown in Table 1. The percentage of missing answers (or "Cannot decide" option) was always lower than 5\%, and there was no ceiling or floor effect in any of the nine items. The median pre-surgical Catquest-9SF raw score was 27 (23-30), and there was no ceiling or floor effect at the scale level. The reliability was satisfactory, with a Cronbach alpha coefficient of 0.87 .

Table 1 Responses to the nine items of the pre-surgery Catquest-SF9

\begin{tabular}{lllllll}
\hline Item, N (\%) & 1-Very great difficulty & 2-Great difficulty & 3-Some difficulty & 4-No difficulty & Total responses & Missing \\
\hline A - Difficulties in any way in daily life & $44(5)$ & $252(30)$ & $469(55)$ & $83(10)$ & $848(100)$ & $0(0)$ \\
B - Satisfaction with vision ${ }^{\text {}}$ & $102(12)$ & $476(56)$ & $250(30)$ & $20(2)$ & $848(100)$ & $0(0)$ \\
C1 - Reading text in newspaper & $68(8)$ & $232(27)$ & $377(44)$ & $169(20)$ & $846(100)$ & $2(0)$ \\
C2 - Recognizing faces & $24(3)$ & $98(12)$ & $239(28)$ & $483(57)$ & $844(100)$ & $4(0)$ \\
C3 - Seeing prices & $41(5)$ & $191(23)$ & $365(43)$ & $235(28)$ & $832(98)$ & $16(2)$ \\
C4 - Seeing to walk on uneven ground & $18(2)$ & $105(12)$ & $342(40)$ & $379(45)$ & $844(100)$ & $4(0)$ \\
C5 - Seeing to do needlework, etc. & $44(5)$ & $180(21)$ & $364(43)$ & $222(26)$ & $810(96)$ & $38(4)$ \\
C6 - Reading text on TV & $90(10)$ & $253(30)$ & $347(41)$ & $150(18)$ & $840(99)$ & $8(1)$ \\
C7 - Seeing to carry out a preferred hobby & $22(3)$ & $123(14)$ & $342(40)$ & $327(49)$ & $814(96)$ & $34(4)$ \\
\hline
\end{tabular}

Response modalities: 1-Very dissatisfied, 2-Fairly dissatisfied, 3-Fairly satisfied, 4-Very satisfied 


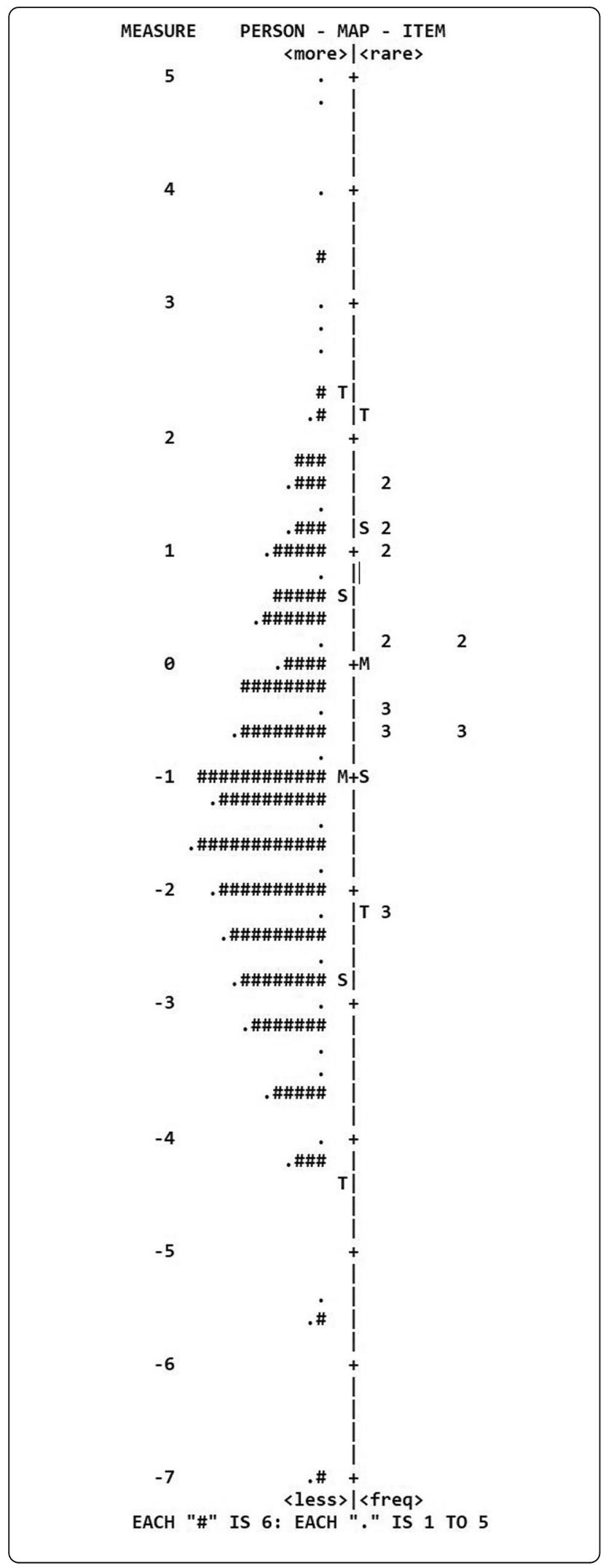

Fig. 4 Person-item map for preoperative data. Items to the right, 2 or 3 signifies the 9 items, and persons to the left, \# signifies 6 persons. $\mathrm{M}$ stands for mean value, $\mathrm{S}$ for 1 standard deviation and $\mathrm{T}$ for 2 standard deviations

The Rasch analysis showed good precision, with a person separation of 2.32 and a person reliability of 0.84 . The category probability curves were ordered for both kinds of response options (A\&C1-7 and B; see Table 1 footnote). Item statistics showed no misfit, meaning no redundancy and no outliers among the items. The fit statistics included infit and outfit mean square, and both values should be close to 1 for the items. In this study, the infit mean square varied between 0.70 and 1.32 and the outfit mean square between 0.74 and 1.25 , thus lying in an ideal range (Table 2).

The observed raw variance explained by the measures was $58 \%$ and the unexplained variance in the first contrast had an eigenvalue of 1.60, indicating a unidimensional questionnaire. Differential item functioning was not observed for gender in any item $(<0.5$ logits in all cases). The targeting (item difficulty versus person ability) for preoperative data showed a mean person Rasch score of -1.12 (item score set to 0 ), indicating that the respondents were more able than the difficulty of the items. This is also shown in the person-item map (Fig. 4).

The Catquest-9SF questionnaire was filled in by 211 patients during the 3 months after surgery (mean number of days after surgery: $26 \pm 21$ days). A clear ceiling effect was present in this post-surgical assessment, with $132(62.6 \%)$ patients showing the highest possible raw score. The median raw score changes from pre- to postsurgical assessment was 8 (5-11) points, and the paired $\mathrm{t}$-test showed a significant difference between the pre-

Table 2 Fit statistics and Rasch measure of item difficulty for the Catquest-9SF items

\begin{tabular}{llll}
\hline Item & $\begin{array}{l}\text { Infit mean } \\
\text { square }\end{array}$ & $\begin{array}{l}\text { Outfit mean } \\
\text { square }\end{array}$ & $\begin{array}{l}\text { Rasch } \\
\text { measure }\end{array}$ \\
\hline $\begin{array}{l}\text { A - Difficulties in any way in } \\
\text { daily life }\end{array}$ & 0.70 & 0.74 & -0.60 \\
$\begin{array}{l}\text { B - Satisfaction with vision } \\
\text { C1 - Reading text in the }\end{array}$ & 1.00 & 1.00 & -2.21 \\
newspaper & 1.08 & 1.07 & -0.40 \\
C2 - Recognizing faces & 1.32 & 1.25 & 1.59 \\
C3 - Seeing prices & 0.81 & 0.81 & 0.13 \\
$\begin{array}{l}\text { C4 - Seeing to walk on } \\
\text { uneven ground }\end{array}$ & 1.14 & 1.12 & 1.18 \\
$\begin{array}{l}\text { C5 - Seeing to do } \\
\text { needlework etc. }\end{array}$ & 0.96 & 0.94 & 0.12 \\
C6 - Reading text on TV & 1.14 & 1.13 & -0.70 \\
C7 - Seeing to carry out a & 0.94 & 0.87 & 0.88 \\
preferred hobby & & & \\
\hline
\end{tabular}




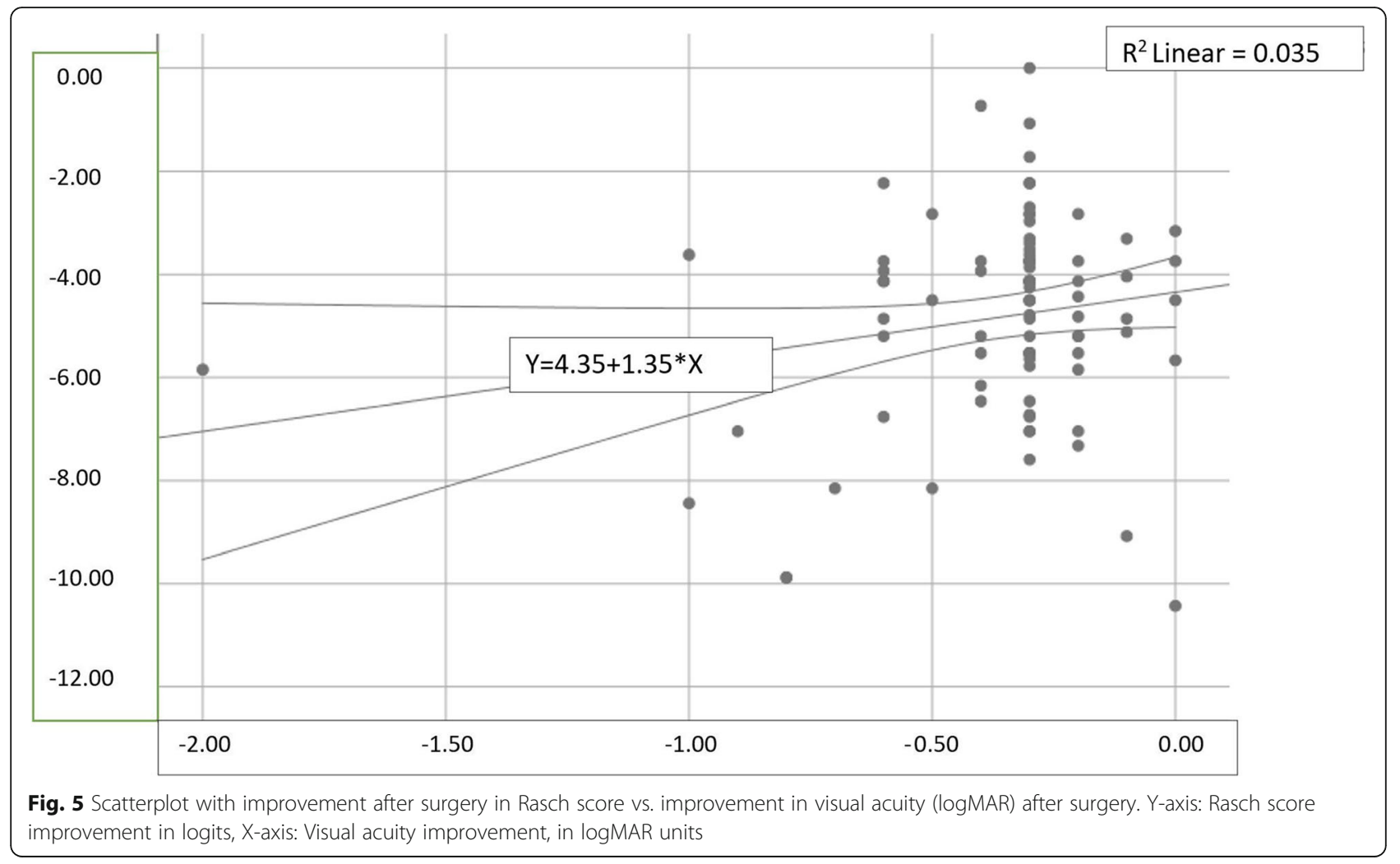

and post-surgical raw scores $(P<0.0001)$. Rasch analysis of the 211 completed pre and postoperative questionnaires showed a large improvement in the average person Rasch score, from $-1.16 \pm 1.73$ logits to $-5.72 \pm 1.24$ logits. The improvement was on average $4.56 \pm 2.02$ logits. The effect size calculated as improvement/standard deviation for baseline was 2.6. The improvement in Rasch score after surgery vs. the improvement in visual acuity (logMAR notation) is demonstrated in a scatter plot (Fig. 5).

\section{Discussion}

In this study, the nine items of the French version of Catquest-9SF completed using an electronic notepad were shown to be Rasch homogenous and to have good psychometric properties. The Cronbach alpha coefficient and the person separation and person reliability indices were congruent and indicated an adequate reliability for the entire scale. The questionnaire was also found to be sensitive to change. As shown in Fig. 4, there was a relationship between improvement in Rasch score (logits) and improvement in visual acuity (logMAR) after surgery.

In daily clinical practice, scientific evidence is lacking in identifying patients who are likely to significantly benefit from cataract surgery [25]. The information from Catquest-9SF can be valuable during patient consultations as it helps to determine the optimal time of intervention [26]. It may also help to explain the progression of the disease and to manage the patient's expectations [27]. The data generated by Catquest-9SF have clinical value since they can be discussed by the physician and the patient in choosing or adapting the care pathway.

The digital collection of Catquest-9SF data prior to consultations for patients with cataracts is implemented in routine practice in the two French ophthalmologic centres participating in this study (Institut Ophtalmologique Sourdille-Atlantique in Nantes and Chénieux Ophtalmologie in Limoges). There are many advantages to using notepads for data collection as these bypass the time-consuming step of transferring the data from paper to computer and makes it easy to improve the ergonomics. In both centres, patients completed the questionnaire in the waiting room using a notepad installed on a mobile table-top set which could be adjusted to the patient's eye-level. Some elderly patients had found it difficult to click and move through the questions because they were using their nail on the touch screen, which caused them to click several times when entering an answer, causing mistakes and frustration. To improve the ergonomics, the table-top set was lowered, and the angle of the touch screen adjusted to facilitate clicking, which resulted in improved data quality and response rate. The electronic setup is also appropriate for use by clinicians as it presents each item's raw score (not the Rasch 
analysed score) change from pre- to post-surgery using a spider chart. This simple dashboard with an ergonomic layout was found to be suitable for use in routine care.

Further work is needed to improve and adjust this layout and make it legible for patients. Another issue may be that being asked to complete the questionnaire before a decision is taken regarding surgery might tempt the patient to exaggerate their symptoms to get surgery. It is therefore important to ask for honest reporting if Catquest-9SF is to be used in establishing indications for surgery.

One weakness in the present study is the handling of postoperative questionnaires. The electronic notepads were situated at the participating clinics, indicating that the postoperative questionnaires could only be completed by those patients who made a postoperative clinic visit. This might have introduced a bias concerning follow-up data. Moreover, the answers given by patients completing the postoperative questionnaire at the clinic may have been influenced by a wish to please the surgeon.

This study has shown that Catquest-9SF is a suitable measure of subjective visual functioning in a French cataract population in France. The questionnaire was successfully translated into French and demonstrated robust psychometric properties. It is therefore suitable for use in French patients with cataract and following cataract surgery. Applicability in daily clinical practice may be improved without loss of accuracy by using a digital version of the questionnaire. This digital format alleviates the burden of data collection, improves data quality, and facilitates real-time discussions between practitioners and patients during consultation.

\section{Conclusions}

A French version of the Catquest-9SF was presented and showed good psychometric properties. This version is suitable for use in French-speaking patients. The French version of Catquest-9SF was presented to patients through an electronic notepad. The electronic format of the Catquest-9SF was convenient for patients and showed good validity.

\section{Supplementary Information}

The online version contains supplementary material available at https://doi. org/10.1186/s40662-021-00233-7.

Additional file 1. Patient workflow with the digital version of Catquest9SF.

\section{Acknowledgements}

Not applicable.

\section{Authors' contributions}

GK was a major contributor in writing the manuscript. ML and AR performed the statistical calculations. FL, TM, and MW made the data available. All authors read and approved the final manuscript.

\section{Funding}

This study was financed by the Consortium VBHC, France. The funding source had no role in the design and conduct of the study; the collection, management, analysis, or interpretation of the data; the preparation, review, or approval of the manuscript; or the decision to submit the manuscript for publication.

\section{Availability of data and materials}

The datasets generated and analysed during the current study are not publicly available due to integrity issues but are available from the corresponding author on reasonable request.

\section{Declarations}

Ethics approval and consent to participate

According to the French regulation, the calibration of a PROM instrument falls into a specific research category that does not require the approval of an ethics committee, but a formal declaration to the French Data Protection Authority (CNIL) which was officially recorded on August 17, 2017.(1) The regulatory procedure that applies to this research category requires (i) a detailed patient information about the research and (ii) a non-opposition statement from the patient in order to use personal data for scientific purpose. This two-step process was secured through the digital platform where patient's information and non-opposition were automatically administered before granting access to the Catquest-9SF questionnaire. Data pseudonymization was also automatically performed to comply with General Data Protection Regulation. (1) The CNIL declaration was registered under the number \#2219673.

\section{Consent for publication}

Not applicable.

\section{Competing interests}

The authors declare that they have no competing interests.

\section{Author details}

${ }^{1}$ Chair of Innovation \& Value in Health, University of Paris School of Medicine, Paris, France. ${ }^{2}$ Value-Based Health Care Consortium, Paris, France.

${ }^{3}$ Prom-Time, Paris, France. ${ }^{4}$ Service de Santé Publique et d'Épidémiologie, AP-HP Paris-Saclay, Le Kremlin-Bicêtre, France. ${ }^{5}$ Centre de recherche en Épidémiologie et Santé des Populations, Inserm, Université Paris-Saclay, Villejuif, France. ${ }^{6}$ Ophtalmologie Chénieux, Polyclinique de Limoges, Limoges, France. ${ }^{7}$ Institut Ophtalmologique Sourdille-Atlantique, Elsan Santé Atlantique, Nantes, France. ${ }^{8}$ Ophthalmology Department, Centre Hospitalier-Universitaire de Nantes, Nantes, France. ${ }^{9}$ Department of Clinical Sciences, Ophthalmology, Faculty of Medicine, Lund University, Lund, Sweden.

Received: 5 September 2020 Accepted: 2 March 2021

Published online: 01 April 2021

\section{References}

1. Lundström M, Dickman M, Henry Y, Manning S, Rosen P, Tassignon MJ, et al. Changing practice patterns in European cataract surgery as reflected in the European Registry of Quality Outcomes for Cataract and Refractive Surgery 2008 - 2017. J Cataract Refract Surg. 2020. https://doi.org/10.1097/j. jcrs.0000000000000457. Online ahead of print.

2. Kirwan C, Nolan JM, Stack J, Moore TC, Beatty S. Determinants of patient satisfaction and function related to vision following cataract surgery in eyes with no visually consequential ocular co-morbidity. Graefes Arch Clin Exp Ophtalmol. 2015;253(10):1735-44

3. Ribeiro F, Cochener B, Kohnen T, Mencucci R, Katz G, Lundström M, et al. Definition and clinical relevance of the concept of functional vision in cataract surgery ESCRS Position Statement on Intermediate Vision. J Cataract Refract Surg. 2020;46(Suppl 1):S1-3.

4. OECD. Health at a glance 2019: OECD indicators. Paris: OECD Publishing; 2019. https://doi.org/10.1787/4dd50c09-en.

5. Khadka J, McAlinden C, Pesudovs K. Quality assessment of ophtalmic questionnaires: review and recommendations. Optom Vis Sci. 2013;90(8): 720-44. 
6. Lundström M, Pesudovs K. Catquest-9SF patient outcomes questionnaire: nine-item short-form Rasch-scaled revision of the Catquest questionnaire. J Cataract Refract Surg. 2009;35(3):504-13.

7. McAlinden C, Gothwal VK, Khadka J, Wright TA, Lamoureux EL. Pesudovs K A head-to-head comparison of 16 cataract surgery outcome questionnaires. Ophthalmology. 2011;118(12):2374-81.

8. Lundström M, Barry P, Henry Y, Rosen P, Stenevi U. Evidence-based guidelines for cataract surgery: guidelines based on data in the European Regsitry of Quality Outcomes for Cataract and Refractive Surgery database. J Cataract Refract Surg. 2012;38(6):1086-93.

9. Mahmud I, Kelley T, Stowel C, Haripriya A, Boman A, Kossler I, et al. A proposed minimum standard set of outcome measures for cataract surgery. JAMA Ophtalmol. 2015;133(11):1247-52.

10. Lundström M, Behndig A, Kugelberg M, Montan P, Stenevi U, Pesudovs K. The outcome of cataract surgery measured with the Catquest-9SF. Acta Ophthalmol. 2011;89(8):718-23.

11. Gothwal VK, Wright TA, Lamoureux EL, Lundström M, Pesudovs K. Catquest questionnaire: re-validation in an Australian cataract population. Clin Exp Ophthalmol. 2009;37(8):785-94.

12. Harrer A, Gerstmeyer K, Hirnschall N, Pesudovs K, Lundström M, Findl O, Impact of bilateral cataract surgery on vision-related activity limitations. J Cataract Refract Surg. 2013;39(5):680-5.

13. Skiadaresi E, Ravalico G, Polizzi S, Lundström M, González-Andrades $M$, McAlinden C. The Italian Catquest-9SF cataract questionnaire: translation, validation and application. Eye Vis (Lond). 2016;3:12.

14. Lundström M, Llovet F, Llovet A, Martinez del Pozo M, Mompean B, González JV, et al. Validation of the Spanish Catquest-9SF in patients with a monofocal or trifocal intraocular lens. J Cataract Refract Surg. 2016;42(12): $1791-6$

15. Visser MS, Dieleman M, Klijn S, Timman R, Lundström M, Busschbach JJV, et al. Validation, test-retest reliability and norm scores for the Dutch Catquest-9SF. Acta Ophthalmol. 2017;95(3):312-9.

16. Nielsen E, Lundström M, Pesudovs K, Hjortdal J. Validation of Catquest-9SF in Danish: developing a revised form of the Catquest-9SF - the Danish Catquest-7SF. Acta Ophthalmol. 2019;97(2):173-7.

17. Lin X, Li M, Wang M, Zuo Y, Zhu S, Zheng Y, et al. Validation of Catquest9SF questionnaire in a Chinese cataract population. PLoS One. 2014;9(8): e103860.

18. Wild D, Grove A, Martin M, Eremenco S, McElroy S, Verjee-Lorenz A, et al. Principles of Good Practice for the Translation and Cultural Adaptation Process for Patient-Reported Outcomes (PRO) Measures: report of the ISPOR Task Force for Translation and Cultural Adaptation. Value Health. 2005;8(2): 94-104.

19. StataCorp LP. Stata statistical software: release 12.1. College Station: StataCorp LP; 2012.

20. de Vet HCW, Terwee CB, Mokkink LB, Knol DL. Measurement in Medicine: Cambridge University Press; 2011. https://doi.org/10.1017/CBO9780511 996214

21. Terwee CB, Bot SD, de Boer MR, van der Windt DA, Knol DL, Dekker J, et al. Quality criteria were proposed for measurement properties of health status questionnaires. J Clin Epidemiol. 2007;60(1):34-42.

22. Gadermann AM, Guhn M, Zumbo BD. Estimating ordinal reliability for Likerttype and ordinal item response data: a conceptual, empirical, and practical guide. Pract Assessment Res Eval. 2012;17:3.

23. Bland JM, Altman DG. Statistics notes: Cronbach's alpha. BMJ. 1997; 314(7080):572. https://doi.org/10.1136/bmj.314.7080.572.

24. Bond TG, Fox CM. Applying the Rasch model: fundamental measurement in the human sciences. 2nd ed. New York: Routledge, Taylor \& Francis Group; 2007.

25. Kessel L, Andresen J, Erngaard D, Flesner P, Tendal B, Hjortdal J. Indication for cataract surgery. Do we have evidence of who will benefit from surgery? A systematic review and meta-analysis. Acta Ophthalmol. 2016;94(1):10-20.

26. Lundström M, Stenevi U. Analyzing patient-reported outcomes to improve cataract care. Optom Vis Sci. 2013;90(8):754-9.

27. Lundström M, Albrecht S, Håkansson I, Lorefors R, Ohlsson S, Polland W, et al. NIKE: a new clinical tool for establishing levels of indications for cataract surgery. Acta Ophtalmol Scand. 2006;84(4):495-501.

\section{Ready to submit your research? Choose BMC and benefit from:}

- fast, convenient online submission

- thorough peer review by experienced researchers in your field

- rapid publication on acceptance

- support for research data, including large and complex data types

- gold Open Access which fosters wider collaboration and increased citations

- maximum visibility for your research: over $100 \mathrm{M}$ website views per year

At $\mathrm{BMC}$, research is always in progress.

Learn more biomedcentral.com/submissions 